\title{
A Pediatric Case of Parotid Manifestation of Recklinghausen Neurofibromatosis
}

\section{Haidar' ${ }^{1}$, J. Ziani ${ }^{2}$, A. Charai ${ }^{1}$, M. Bennani ${ }^{2}$, D. Kamal1, H. Baybay ${ }^{2}$, M. N. Elamine elalami ${ }^{1}$, F. Z. Mernissi ${ }^{2}$}

${ }^{1}$ Department of Otolaryngology, Hassan II Hospital University, Fez, Morocco

${ }^{2}$ Department of Dermatology, Hassan II Hospital University, Fez, Morocco

*Corresponding author: Haidar Maojil, Departement of Otolarygology, Hassan II Hospital University, Fez, Morocco

Received Date: March 07, 2020; Accepted Date: March 16, 2020; Published Date: March $23,2020$.

Citation: Haidar H, Ziani J, Charai1 A, Rasso A, Kamal D. (2020) A Pediatric Case of Parotid Manifestation of Recklinghausen Neurofibromatosis. Journal of Clinical Case Reports and Studies, 1(2): Doi: 10.31579/ 2690-8808/007

Copyright: (C) 2020. Jihane Ziani. This is an open-access article distributed under the terms of the Creative Commons Attribution License, which permits unrestricted use, distribution, and reproduction in any medium, provided the original author and source are credited.

\section{Case Report}

A 12-year-old girl is refered to our ORL department. The disease started in childhood with the appearance of multiple hyper pigmented skin macules. Dermatological exam showed of hyper pigmented skin macules on the chest and back, multiple pigmented spots with diameter $>1.5 \mathrm{~cm}$ (Figure 1), axillary freckling.
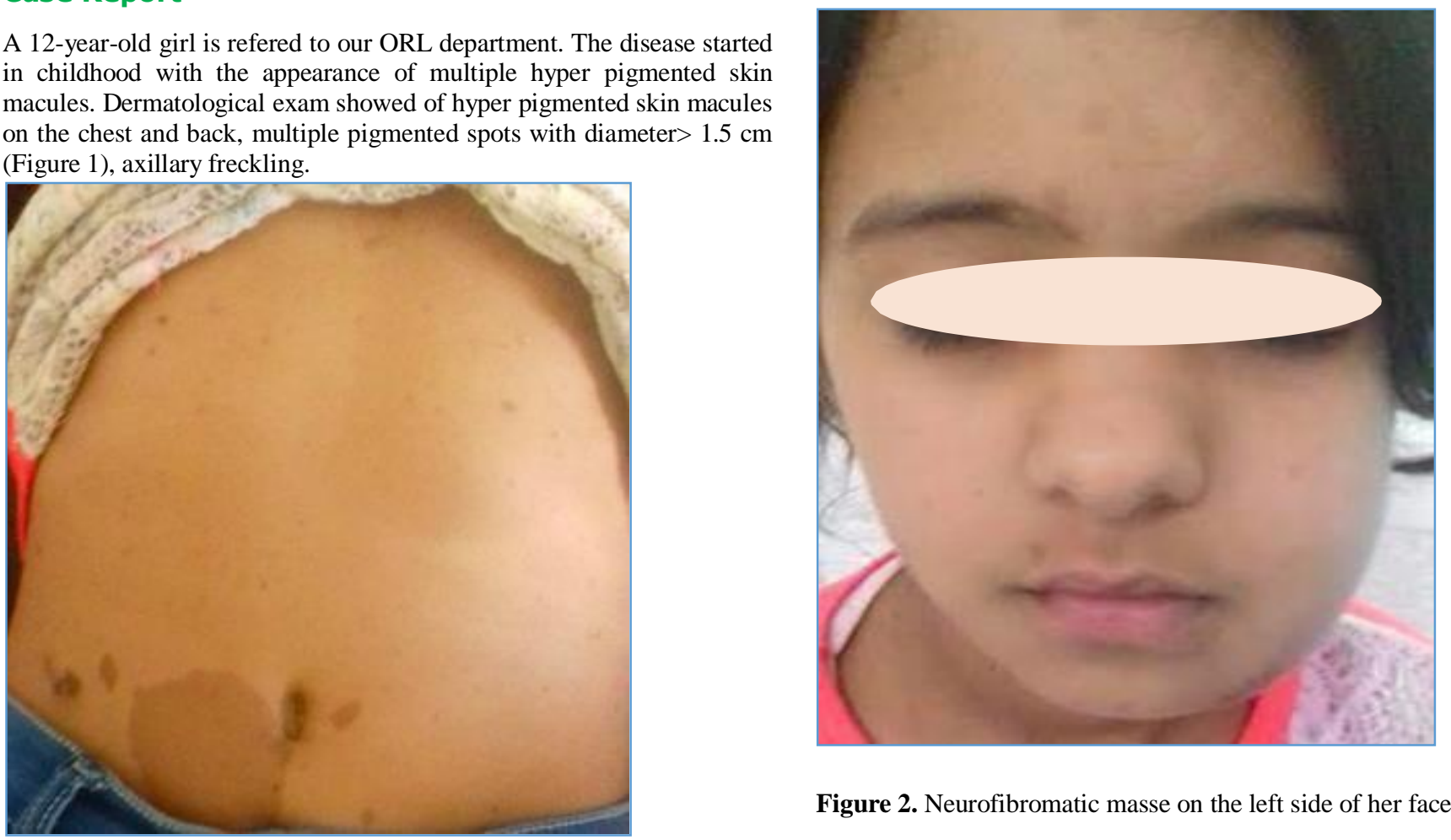

Figure 2. Neurofibromatic masse on the left side of her face.

Figure 1. Multiple hyper pigmented skin macules pour la plus grande mesurant $7 \mathrm{~cm}$.

During oral examination, we saw a formation of neurofibromatic mass on the left side of her face (Figure 2) mucous membranes were not affected.

MRI showed the presence of a hypodense left intraparotideal process before and after injection of contrast medium, molding the intraparotidic vascular structure and extending along the facial vascular bundle from the mandibular homolateral angle below, and the region pretrageal at the top, with significant narrowing of the external auditory canal of the homolateral ear. 
Neurofibromatosis are genetic diseases that predispose to the development of tumors in the nervous system. There are two essential forms: neurofibromatosis type 1 and neurofibromatosis type 2. This disease cannot be treated. Only complications are treated. The parotidojugual neurofibroma is a readily unique lesion, centered on the parotid region and more or less invading the jugular region. The parotid tissue is invaded by the neurofibroma and the position of the facial nerve is unpredictable, the neurofibroma can be developed from the facial nerve. We can also find a posterior tilt of the pavilion of the ear and occipital osteolytic lesions. Endo-oral extensions should be systematically sought [1].

Bone deformities of the upper and lower jaw are clearly associated with the presence of facial neurofibromas that invade the branches of the trigeminal. A progressive widening of the superior orbital fissure and the oval foramen. The mandible on the affected side is asymmetrical just as the adjacent part of the maxilla and the external wall of the orbit are destroyed [2]. A parotid swelling that can reflect a nervous attack, while thinking of the facial nerve even if its involvement is rare (0.5 to $1 \%)$ [3], and studies, as far as possible, its path in front of any tumor in the region parotid. Imaging plays an important role in positive diagnosis, prognostic evaluation, price orientation in therapeutic charge and progressive followup of lesions. Treatment is surgical but abstention and monitoring may be indicated because the progress is slow.

The particularity of our observation is that it a rare presentation of the disease in children as well as the interest of imagery to evoke the diagnosis of parotid neurofibroma and assessment of locoregional extension and the follow-up of these tumors.

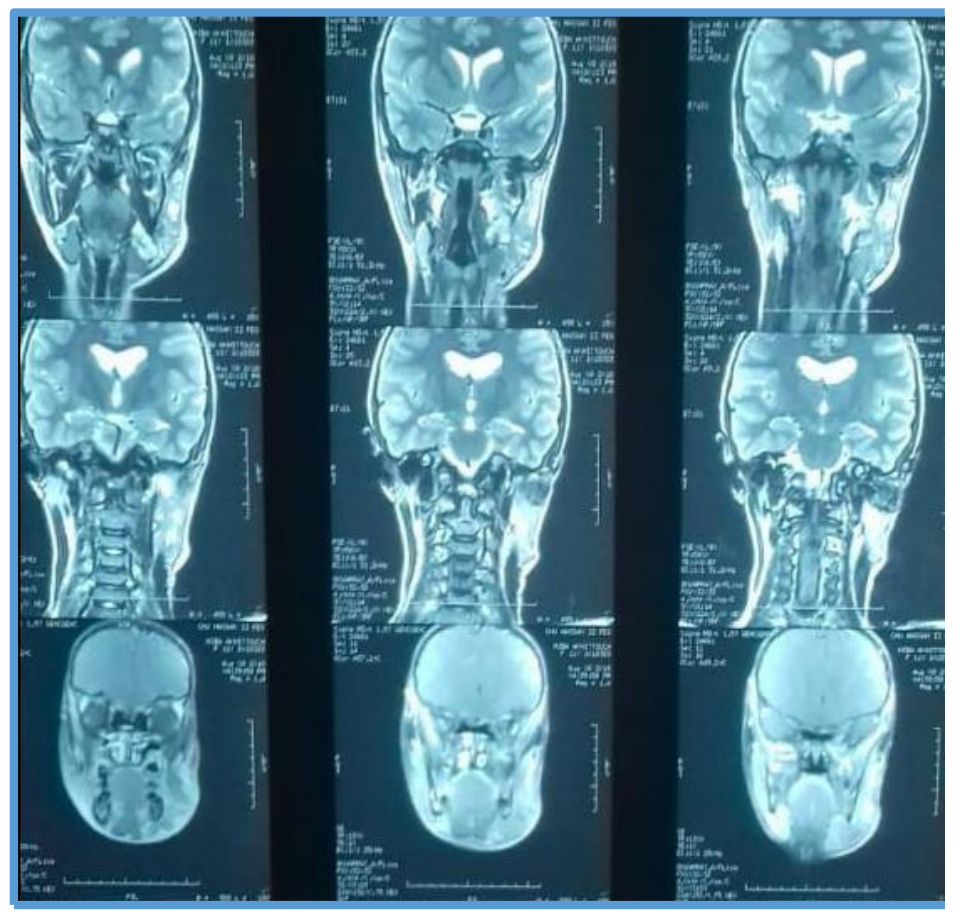

Figure 3. Expanding left parotid process with partial agenesis of the large left sphenoid wing.
Conflicts of interest: Author declares that there is no conflict of interest

\section{References}

1. Jayachandran D, Sunantha S, Gopalaiah H, Veeraraghavan G. (2014). Plexiform neurofibromatosis involving face and oral cavity. J Oral Maxillofac Pathol. 18:114-117

2 Friedrich R, Heiland M, Kehler U, Schmelzle R. (2003). Reconstruction of Sphenoid Wing Dysplasia with Pulsating Exophthalmos in a Case of Neurofibromatosis Type 1 Supported by Intraoperative Navigation Using a New Skull Reference System. Skull Base. 13:211-217

3. Neurofibromatosis. Conference statement. National Institutes of Health Consensus Development Conference. Arch Neurol. (1988). 45:575-578 